\title{
Prognostic Value of Long QT Interval in Acute and Severe Organophosphate Poisoning
}

\author{
Shahin Shadnia, MD, PhDa, Arash Okazia, Navid Akhlaghia, Ghazal Sasanian ${ }^{b}$, Mohammad \\ Abdollahib \\ aLoghman-Hakim Hospital Poison Center, Faculty of Medicine and Toxicological Research Center, Shahid Beheshti University of \\ Medical Sciences, Tehran, Iran \\ bFaculty of Pharmacy, and Pharmaceutical Sciences Research Center, Tehran University of Medical Sciences, Tehran, Iran
}

\begin{abstract}
Introduction: Suicidal poisoning with organophosphorus (OP) pesticides is common, particularly from rural areas. This highlights the importance of determining an OP poisoning prognosis to decide how aggressive treatment should be. There are reports suggesting a relationship between prolonged corrected QT (QTC) interval and the severity of poisoning. We aimed to evaluate the prognostic utility of this clinical tool in OP poisoning (OPP) patients.

Methods: Patients with the primary diagnosis of OPP who were admitted to the intensive care unit (ICU) of Loghman-Hakim Hospital Poison Centre (LHHPC) were the subjects of this prospective study. Cholinesterase (CE) activity and the QTC interval was determined for each patient using the Bazett formula and considering $<440 \mathrm{msec}$ as normal. Comparative outcomes of the study were duration of both hospitalization and mechanical ventilation, serum CE activity on admission and its daily level, total amount of atropine administered, analysis of the QT and QTC intervals in the primary ECG on admission and at the end of hospitalization, and rate of mortality.

Results: The study included 42 patients with a diagnosis of OPP. The mean age of the patients was 32, ranged from 12 to 81 years old. The mortality rate was 37.5\%. There was no significant difference between two groups (prolonged and normal QTC intervals) according to gender and age ( $p=.491$ and $p=.133$, respectively). The CE level for long and normal QTC interval groups was $3.90 \pm 0.33$ $\mathrm{kU} / \mathrm{L}$ vs. $4.41 \pm 0.23 \mathrm{kU} / \mathrm{L}$, respectively. The mortality rate in the long QTC group was significantly higher than that of the normal QTC group $(p=.044)$. Moreover, the average period of hospitalization in patients with prolonged QTC interval was higher than the other group $(p=.02)$. The average atropine required to control the muscarinic signs and symptoms such as salivation, bronchorrehea, and miosis in patients with prolonged QTC interval was $38.60 \mathrm{mg}$; in patients with normal QTC interval it was $20.02 \mathrm{mg}(p=.013)$.
\end{abstract}

Conclusion: QTC interval prolongation may have prognostic value in OPP.

\section{INTRODUCTION}

Organophosphate compounds (OPs) are one of the most common pesticides used to control agricultural and indoor pests. Gunnell et al. [1] conducted a systematic review of the worldwide literature to estimate the number of pesticide suicides in each of the WHO's 6 regions and the global burden of fatal self-poisoning with pesticides. They estimated 258,234 (plausible range 233,997-325,907)

Keywords: organophosphate, pesticide, poisoning, QT interval

Acknowledgment: The authors wish to thank Dr. L.S. Nelson from the New York City Poison Control Center for reading and editing the paper, and the nurses of Loghman-Hakim Hospital Poison Center, especially Mrs. S. Bana-Jafari and Mrs. M. Rezvani, for their assistance.

Notes: There was no outside funding of any kind used for this study.

The authors have no potential financial conflicts of interest to report.

Corresponding author: Professor Mohammad Abdollahi, Faculty of Pharmacy, and Pharmaceutical Sciences Research Center, Tehran University of Medical Sciences, Tehran 1417614411, Iran. Email: mohammad.abdollahi@utoronto.ca 
deaths from pesticide self-poisoning annually, accounting for $30 \%$ (range $27-37 \%$ ) of suicides globally. The proportion of all suicides using pesticides varies from $4 \%$ in the European region to $>50 \%$ in the Western Pacific region. It is estimated that there are 34,000 suicides annually in the Middle East region, and 20\% of suicides in the Middle East region are the result of pesticide ingestion [1].

In Iran, organophosphate poisoning (OPP) is a major health problem [2-5], and OPs are the main cause of pesticide poisoning and pesticide-related deaths in Tehran [6-10].

The mortality rate is between $2-30 \%$ despite appropriate treatment [9-11]. Therefore, the prediction of mortality is important to allow proper resource allocation and therapeutic aggressiveness. Although the plasma or urine OP concentrations, cholinesterase (CE) activity, and serum ß-glucuronidase level correlate with severity and mortality rates [12-18], these laboratory methods are not readily available and their usefulness remains controversial [19]. The absence of specific laboratory methods indicates the need for a clinically-based prognostic system [20].

Studies on the cardiac effects of OPP have been inconclusive, and sometimes conflicting results have been reported. A relationship between a prolonged corrected QT (QTC) interval on the electrocardiogram (ECG) of patients with OPP and the severity of poisoning has been reported previously to assist in determining prognosis and therapy [21].

In the current study, we aimed to investigate the relationship between the QTC interval and both the severity and prognosis of OPP.

\section{MATERIALS AND METHODS}

This prospective case-control study included all patients who were admitted to the ICU of the Loghman-Hakim Hospital Poison Centre (LHHPC) for 1 year. The study was approved by the IRB and each patient's relative gave written informed consent.

The diagnosis for all patients was OPP. Establishment of the diagnosis in all cases was based on the history taken from the patients' relatives, characteristic odor of the gastric contents, and other circumstantial evidence, such as availability of a poison bottle or a label found by the relatives, cholinergic clinical features that resolved after atropinization, and decrease by $>25 \%$ from normal in serum CE activity on admission (normal range >1900-2500 kU/L). Patients with diabetes mellitus; cardiovascular, respiratory, renal and/or hepatic failure; surgical operation in the past 7 days; positive urine for conventionally-abused drugs; co-ingestion with other agents; loss of consciousness at the time of admission (Glasgow Coma Scale $<12$ ), which was responsive to coma cocktails; and advanced medical management (treatments such as gastrointestinal decontamination, administration of atropine, oxime, and intubation and/or ventilation) for OPP in any medical center before admission in LHHPC were excluded.

All patients received gastrointestinal decontamination in the first 6 hours after the onset of poisoning, and oral activated char- coal was administered in the emergency department (ED). All patients were treated with atropine, pralidoxime, and adequate hydration under supervision of the same group of physicians and received equivalent nursing services by the same ICU personnel. All patients received endotracheal intubation and mechanical ventilation.

Information for all patients-including gender, age, reason of poisoning, route of exposure, duration of both hospitalization and mechanical ventilation, serum CE activity on admission and daily level, total amount of atropine administered, QT and QTC interval on the ECG on admission and at the end of hospitalization, and outcome-were collected and recorded. The QTC interval for all cases was determined by use of the Bazett formula (QTC $=$ QT/vRR) [22]. Calculation was done by physicians and by calculator and not by ECG machine. Based on the length of QTC interval, cases were divided into two groups of prolonged and normal QTC interval. By definition, the QTC interval was considered prolonged if it was more than 0.44 seconds. To determine the relationships among the collected data, the Chi Square and the Student t-test were applied and a $p$ value $<.05$ was considered statistically significant.

\section{RESULTS}

Of 42 included cases, $47.6 \%(n=20)$ were male and $52.4 \%(n=$ 22 ) were female. The age range was $12-81$ years, with a mean age of $32 \pm 16.3$ years, and a median of 24 years (Table 1 ). Except for 1 case, all cases were suicide attempts. The mortality rate was $35.7 \%, 66.7 \%(n=10)$ of whom were male and 33.3\% $(n=5)$ female. The average hospitalization period was $10.1 \pm 6.7$ days and the daily average of atropine administered was $30.9 \pm$ $23.25 \mathrm{mg}$.

As shown in Table 2, 25 (59.5\%) patients showed a prolonged QTC interval $(501.6 \pm 14.34 \mathrm{msec})$ while $40.5 \%(\mathrm{n}=17)$ had a normal QTC interval $(395.88 \pm 10.04 \mathrm{msec})$. There was no significant difference between the two groups according to sex and age $(p=.491$ and $p=.133$, respectively). The mortality rate in the patients with a prolonged QTC interval was higher than that of the patients with a normal QTC interval $(p=.044)$. The average length of hospitalization was greater (7.4 days) in patients with prolonged QTC interval than the other group (5.24 days) $(p=.02)$. The use of mechanical ventilation was not different between the two groups $(p=.056)$.

$\begin{aligned} & \text { Table 1: Distribution of Patients Who Attempted } \\
& \text { Suicide by OPP According to Age, }\end{aligned}$
\begin{tabular}{lcc}
\hline Age Group (Years) & Number & Percent \\
\hline $\mathbf{1 2 - 2 0}$ & 10 & 23.8 \\
\hline $\mathbf{2 1 - 4 0}$ & 22 & 52.4 \\
\hline $\mathbf{4 1 - 6 0}$ & 6 & 14.3 \\
\hline $\mathbf{6 0}$ & 4 & 9.5 \\
\hline
\end{tabular}




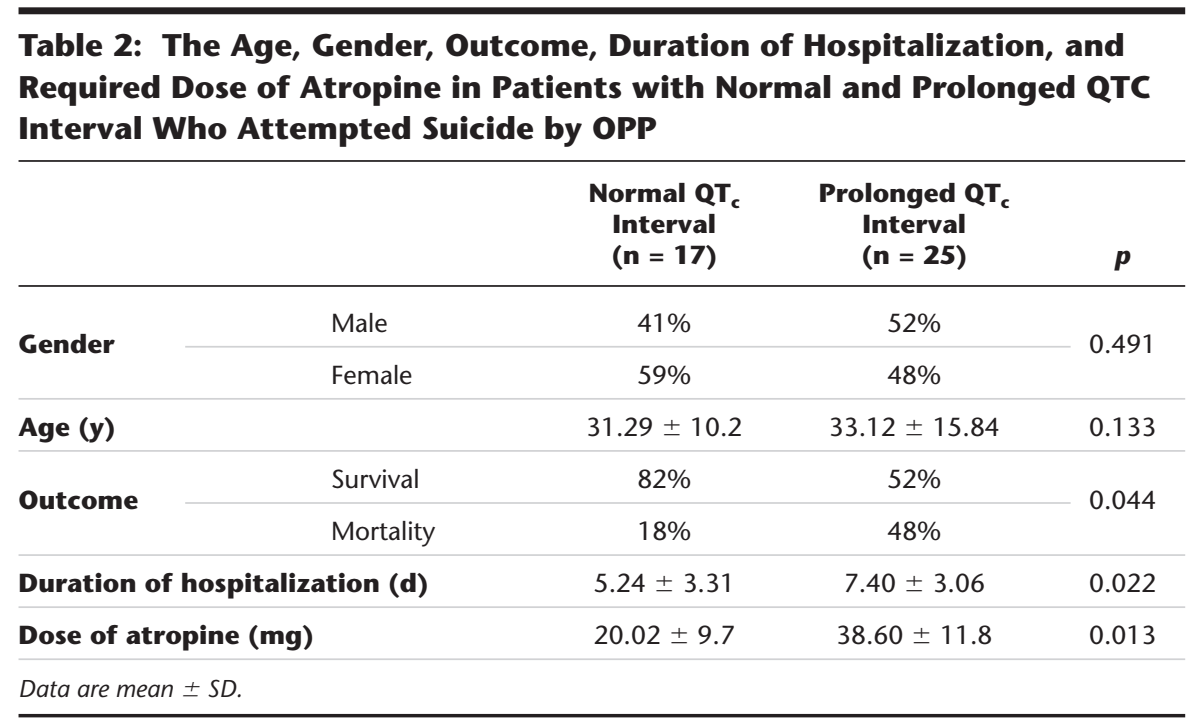

The average dose of atropine required to control the symptoms was $38.6 \mathrm{mg}$ in patients with prolonged QTC interval and $20.02 \mathrm{mg}$ in patients with normal QTC interval $(p=.01$, Table 2$)$.

To examine the role of variables on survival, the patients were divided into survival and nonsurvival groups. There was no significant survival difference between the two groups based on gender $(p=.06)$ and age $(29.5 \pm 10.5$ years vs. $37.6 \pm 17.4$ years; $p=.07)$. There was a statistically significant inverse relationship between the survival and nonsurvival groups according to dose of atropine administered during hospitalization $(23 \pm 18.3 \mathrm{mg}$ vs. $48.9 \pm$ $24.79 \mathrm{mg} ; p=.001$ ). There was no significant difference between survival and nonsurvival groups based on measured serum CE activity on admission $(4.84 \pm 1.21 \mathrm{kU} / \mathrm{L}$ vs. $4.51 \pm 1.07 \mathrm{kU} / \mathrm{L}$ respectively; $p=.379$ ).

\section{DISCUSSION}

In the present study, 59.5\% of the OPP cases showed a prolonged QTC interval; these cases had a higher mortality and higher atropine requirement. Based on these findings and due to duration of hospitalization, the present study confirms an association between the prolongation of QTC interval and the intensity of poisoning and prognosis of the patients. No direct relationship between the prolongation of QTC interval and mortality rate in the studied patients $\left(\mathrm{r}^{2}=.096\right)$ was achieved.

ECG abnormalities-such as QT-interval prolongation and polymorphic ventricular tachycardia (e.g., torsades de pointes), ST-T changes, and disturbance of the cardiac conduction system in OPP—have been reported previously [23-28]. The mechanism by which OPs cause cardiotoxic effects is not yet known, but cholinergic innervation of the heart results in both negative chronotropy and negative inotropy that slows myocardial conduction or repolarization [20]. In addition, oxidative stress in the heart cells may be the cause of myocardial damage that may re- sult in several conduction problems [29]. However, unequal sympathetic stimulation of myocardial cells, and interactions with potassium channels and the $\mathrm{Na}^{+} / \mathrm{Ca}^{2+}$ exchanger in the myocardial cell membrane are probably responsible for the occasional prolonged QTC interval [28].

Corroborating the results of our study, in previous reports on OPP, the prolongation of QTC interval has been noted in up to $43.5 \%$ of poisoned patients, correlating with the rate of mortality and respiratory complications [21]. Grmec et al. [30] showed that in OPP, a longer QTC interval and a lower Glasgow Coma Scale (GCS) score accompany higher numbers of intubations and worse outcomes. In addition, they showed that the two measures of GCS score and QTC interval are equally good predictors of respiratory failure and hospital mortality in patients with OPP, but we did not find the same significant statistical correlation with respiratory complications. This indicates that along with the respiratory problems that can cause death in the initial stages of poisoning, cardiac problems can affect the course of illness and the prognosis of patients with OPP.

Also on the basis of our findings, we cannot suggest measurement of serum CE as the only prognostic factor for determination of severity and outcome.

Considering all factors, we conclude that QTC interval prolongation has a prognostic value in OPP.

\section{REFERENCES}

1. Gunnell D, Eddleston M, Phillips MR, et al. The global distribution of fatal pesticide self-poisoning: Systematic review. BMC Public Health. 2007; 7:357.

2. Afshari R, Majdzadeh R, Balali-Mood M. Pattern of acute poisonings in Mashhad, Iran 1993-2000. J Toxicol/Clin Toxicol. 2004;42:965-975. 
3. Moghadamnia AA, Abdollahi M. An epidemiological study of poisoning in northern Islamic Republic of Iran. East Mediterr Health J. 2002;8:88-94.

4. Shadnia S, Azizi E, Hosseini R, et al. Evaluation of oxidative stress and genotoxicity in organophosphorus insecticide formulators. Hum Exp Toxicol. 2005;24:439-445.

5. Pajoumand A, Shadnia S, Rezaie A, et al. Benefits of magnesium sulfate in the management of acute human poisoning by organophosphorus insecticides. Hum Exp Toxicol. 2004;23: $565-569$.

6. Abdollahi M, Jalali N, Sabzevari O, et al. Pesticide poisoning during an 18-month period (1995-1997) in Tehran, Iran. Irn J Med Sci. 1999;24:77-81.

7. Abdollahi M, Jalali N, Sabzevari O, et al. A retrospective study of poisoning in Tehran. J Toxicol/Clin Toxicol. 1997;35: 387-393.

8. Ranjbar A, Solhi H, Mashayekhi FJ, et al. Oxidative stress in acute human poisoning with organophosphorus insecticides; a case control study. Environ Toxicol Pharmacol. 2005;20:88-91.

9. Ranjbar A, Pasalar P, Abdollahi M. Induction of oxidative stress and acetylcholinesterase inhibition in organophosphorous pesticide manufacturing workers. Hum Exp Toxicol. 2002;21: 179-182.

10. Shadnia S, Esmaily H, Sasanian G, et al. Pattern of acute poisoning in Tehran-Iran in 2003. Hum Exp Toxicol. 2007;26: 753-756.

11. Yamashita M, Yamashita M, Tanaka J, et al. Human mortality in organophosphate poisonings. Vet Hum Toxicol. 1997;39:84-85.

12. Abdullat IM, Battah AH, Hadidi KA. The use of serial measurement of plasma cholinesterase in the management of acute poisoning with organophosphates and carbamates. Forensic Sci Int. 2006;162:126-130.

13. Brahmi N, Mokline A, Kouraichi N, et al. Prognostic value of human erythrocyte acetyl cholinesterase in acute organophosphate poisoning. Am J Emerg Med. 2006;24:822-827.

14. Ciesielki S, Loomis DP, Mims SR. Pesticide exposure cholinesterase depression and symptoms among North Carolina migrant farm workers. Am J Public Health. 1994;84: 446-451.

15. Ellenhorn MJ, Schonwald S, Ordog G, et al. Ellenhorn's Medical Toxicology: Diagnosis and Treatment of Human Poisoning. 2nd ed. Baltimore, Md: Williams and Wilkins; 1998.

16. Dart RC, ed. Medical Toxicology. 3rd ed, Philadelphia, Pa: Lippincott Williams \& Wilkins; 2004.
17. Lee P, Tai DHY. Clinical features of patients with acute organophosphate poisoning requiring intensive care. Intensive Care Med. 2001;27:694-699.

18. Soltaninejad K, Shadnia S, Afkhami-Taghipour M, et al. Blood ß-glucuronidase as a suitable biomarker at acute exposure of severe organophosphorus poisoning in human. Hum Exp Toxicol. 2007;26:963-966.

19. Aygun D, Doganay Z, Altintop L, et al. Serum acetylcholinesterase and prognosis of acute organophosphate poisoning. J Toxicol/Clin Toxicol. 2002;40:903-910.

20. Shadnia S, Darabi D, Pajoumand A, et al. A simplified acute physiology score in the prediction of acute organophosphate poisoning outcome in an intensive care unit. Hum Exp Toxicol. 2007;26:623-627.

21. Chuang FR, Jang SW, Lin JL, et al. QTC prolongation indicates a poor prognosis in patients with organophosphate poisoning. Am J Emerg Med. 1996;14:451-453.

22. Bazett HC. An analysis of the time-relationship of electrocardiogram. Heart. 1920;7:353-370.

23. Karki P, Ansari JA, Bhandary S, et al. Cardiac and electrocardiographical manifestations of acute organophosphate poisoning. Singapore Med J. 2004;45:385-389.

24. Wang MH, Tseng CD, Bair SY. QT interval prolongation and pleomorphic ventricular tachyarrhythmia (torsade de pointes) in organophosphate poisoning: report of a case. Hum Exp Toxicol. 1998;17:587-590.

25. Brill DM, Maisel AS, Prabhu R. Polymorphic ventricular tachycardia and other complex arrhythmias in organophosphate insecticide poisoning. J Electrocardiography. 1984;17: 97-102.

26. Ludomirsky A, Klein HO, Sarelli P, et al. Q-T prolongation and polymorphous ("torsdae de pointes") ventricular arrhythmias associated with organophosphorous insecticide poisoning. Am J Cardiol. 1982;49:1654-1658.

27. Saadeh AM, Farsakh NA, al-Ali MK. Cardiac manifestations of acute carbamate and organophosphate poisoning. Heart. 1997;77:461-464.

28. Goldfrank LR, Flomenbaum NE, Lewin NA, et al., eds. Goldfrank's Toxicologic Emergencies. 7th ed. New York, NY: McGraw-Hill; 2002.

29. Abdollahi M, Ranjbar A, Shadnia S, et al. Pesticides and oxidative stress: a review. Med Sci Monit. 2004;10:RA144-RA147.

30. Grmec S, Mally S, Klemen P. Glasgow coma scale score and QTC Interval in the prognosis of organophosphate poisoning. Acad Emerg Med. 2004;11:925-930. 